\title{
Comparing the efficacy of angiotensin receptor-neprilysin inhibitor and enalapril in acute anterior STEMI patients after primary percutaneous coronary intervention: a prospective randomized trial
}

\author{
Youzheng Dong ${ }^{1}$, Yan $\mathrm{Xu}^{1}$, Congcong Ding ${ }^{1,2}$, Zuozhong $\mathrm{Yu}^{1}$, Zhide $\mathrm{Yu}^{1}$, Xin $\mathrm{Xia}^{1}$, Yang $\mathrm{Chen}^{1}$, \\ Xinghua Jiang ${ }^{1}$ \\ ${ }^{1}$ Department of Cardiovascular Medicine, The Second Affiliated Hospital of Nanchang University, Nanchang, China; ${ }^{2}$ Center for Prevention and \\ Treatment of Cardiovascular Diseases, The Second Affiliated Hospital of Nanchang University, Nanchang, China \\ Contributions: (I) Conception and design: Y Dong, X Jiang; (II) Administrative support: Y Xu; (III) Provision of study materials or patients: C Ding, \\ Z Yu, Z Yu; (IV) Collection and assembly of data: Y Chen, X Xia; (V) Data analysis and interpretation: Y Dong, X Jiang; (VI) Manuscript writing: All \\ authors; (VII) Final approval of manuscript: All authors. \\ Correspondence to: Xinghua Jiang, MD. Department of Cardiovascular Medicine, the Second Affiliated Hospital of Nanchang University, No. 1 of \\ Minde Road, Donghu District, Nanchang 330006, China. Email: xinghua_jiang@163.com.
}

Background: For patients with heart failure (HF), the effect of angiotensin receptor-neprilysin inhibitors (ARNIs, sacubitril/valsartan) on cardiac remodeling has been found to be superior to angiotensin-converting enzyme inhibitors (ACEI). However, little data have described the impact of early-initiation ARNI in patients with acute anterior ST-segment elevation myocardial infarction (STEMI).

Methods: In this prospective, randomized, double-blind, parallel-group trial, we enrolled 131 anterior STEMI patients who were treated with primary percutaneous coronary intervention (PCI) between February 2019 and December 2019. All patients received standard STEMI management and were divided into 2 groups (ARNI/enalapril). Primary efficacy outcomes were the between-group difference in change (from baseline to 4-, 12-, and 24-week) in N-terminal pro-B-type natriuretic peptide (NT-proBNP) concentration, left ventricular ejection fraction (LVEF), and left ventricular end-systolic volumes and end-diastolic volumes (LVESV and LVEDV). Secondary outcomes were determined by a composite of death, reinfarction, outpatient HF or HF hospitalization, malignant arrhythmia, and stroke. Safety outcomes included worsening renal function, hypotension, hyperkalemia, angioedema and cough.

Results: We found that NT-proBNP concentration decreased more in the ARNI group than in the enalapril group [4 weeks: ratio of ARNI vs. enalapril 0.36, $95 \%$ confidence interval (CI): 0.24 to 0.52 , $\mathrm{P}<0.001 ; 12$ weeks: 0.54 , 95\% CI: 0.35 to $0.79, \mathrm{P}<0.001 ; 24$ weeks: $0.53,95 \%$ CI: 0.32 to $0.83, \mathrm{P}<0.001$ ). When compared to the enalapril group, the ARNI group patients had a significant reduction in LVEDV $(\mathrm{P}<0.001)$ and LVESV $(\mathrm{P}<0.001)$, and an improvement in LVEF $(\mathrm{P}=0.011)$ at 24 weeks. Secondary outcomes occurred in 13 participants $(20.3 \%)$ in the ARNI group and 22 participants $(34.4 \%)$ in the enalapril group [hazard ratio (HR), 0.56; 95\% CI: 0.28 to $1.12 ; \mathrm{P}=0.102$ ]. The incidence of outpatient HF or HF hospitalization in the ARNI group was significantly lower than that in the enalapril group (HR, 0.36; $95 \%$ CI: 0.14 to $0.94 ; \mathrm{P}=0.037$ ). There were no significant differences in the safety between the 2 groups.

Conclusions: For patients with acute anterior STEMI undergoing primary PCI, early initiation of ARNI provided significant clinical benefits.

Trial Registration: Chinese Clinical Trial Registry (ChiCTR2100042944) registered on February 1, 2021.

Keywords: Angiotensin receptor-neprilysin inhibitor (ARNI); sacubitril/valsartan; enalapril; segment elevation myocardial infarction (STEMI); cardiac remodeling 
Submitted Jun 24, 2021. Accepted for publication Nov 23, 2021.

doi: $10.21037 / \mathrm{cdt}-21-386$

View this article at: https://dx.doi.org/10.21037/cdt-21-386

\section{Introduction}

Even with the advent of coronary intervention techniques and progress in medication, acute myocardial infarction (AMI) remains the main cause of death from coronary artery disease. Other major adverse cardiovascular event following AMI, such as heart failure (HF) and reinfarction, can also impose a heavy economic burden. Ventricular remodeling plays an important role in the poor prognosis after AMI.

In spite of opening of the infarct-related artery as early as possible and the application of the best medical treatment, about $41.5 \%$ of ST-segment elevation myocardial infarction (STEMI) patients still experience ventricular remodeling at 6 months (1). Ventricular remodeling after AMI is affected by many risk factors, such as gender, age, smoking, obesity, diabetes, hypertension, dyslipidemia, location of MI, infarct size, and the presence of multivessel or chronic total occlusion disease (2-6). Among these factors, anterior wall myocardial infarction has been found to raise the risk of ventricular remodeling 1.9-fold compared with other localizations of the infarct (4).

Sacubitril/valsartan, a combination angiotensin receptor-neprilysin inhibitor (ARNI), is a new singlemolecule treatment composed of valsartan and a neutral endopeptidase (NEP) inhibitor prodrug, sacubitril (1:1 ratio) (7). The pharmacological effect of sacubitril/valsartan is achieved by inhibiting the enkephalinase and angiotensin II type 1 (AT1) receptor at the same time, thereby exerting an anti-cardiac remodeling effect. Previous clinical trials have confirmed that sacubitril/valsartan is superior to angiotensin-converting enzyme inhibitors (ACEI) in improving cardiac structural and functional parameters and reducing the risk of HF rehospitalization and cardiovascular death in HF patients with reduced ejection fraction $(8,9)$. In research on MI, several animal experiments have also demonstrated that when compared with enalapril, ARNI therapy could attenuate the scar area after infarction and improve left ventricular (LV) systolic function (10-12). Moreover, in the recent PARADISE-MI trial study (13), the main result adjudicated by the clinical endpoint committee (CEC) showed that, when compared with ramipril, ARNI significantly reduced total (first and recurrent) primary endpoint events by $21 \%$ [rate ratio $0.79 ; 95 \%$ confidence interval (CI): 0.65-0.97; $\mathrm{P}=0.02$ ].

Given that ARNI is superior to ACEI in anti-cardiac remodeling, and may therefore help delay the progression of HF after AMI, we undertook this trial to assess the safety and efficacy of immediate ARNI initiation in patients with acute anterior STEMI after primary percutaneous coronary intervention (PCI). We present the following article in accordance with the CONSORT reporting checklist (available at https://cdt.amegroups.com/article/ view/10.21037/cdt-21-386/rc).

\section{Methods}

\section{Study population}

We conducted a single-center, prospective, randomized, double-blind and parallel-group study. The protocol was approved by the Ethics Committee of the Second Affiliated Hospital of Nanchang University (No. 2019005), and informed consent was taken from all the patients. All methods were performed in accordance with the principles of the Declaration of Helsinki (as revised in 2013) and the Ethical Guidelines for Medical and Health Research Involving Human Subjects. Patients were screened by 2 researchers according to inclusion and exclusion criteria. The main eligibility criteria included the following: (I) participants aged $\geq 18$ years; (II) typical history of chest pain and diagnosed with acute anterior STEMI based on admission electrocardiogram, serum markers of myocardial injury, and invasive coronary angiography; (III) systolic blood pressure $\geq 100 \mathrm{mmHg}$ without symptoms of dizziness/ vertigo during the last 12 hours before randomization; and (IV) provision of informed consent. Patients were excluded based on the following criteria: (I) previous history of MI or chronic HF; (II) previous revascularization (PCI, coronary artery bypass graft, $\mathrm{CABG}$ ), implantation of ventricular assist devices, or heart transplantation; (III) estimated glomerular filtration rate $(\mathrm{eGFR})<30 \mathrm{~mL} / \mathrm{min} / 1.73 \mathrm{~m}^{2}$ or serum potassium $>5.2 \mathrm{mmol} / \mathrm{L}$ at randomization; (IV) known history of liver disease (acute and chronic hepatic impairment, liver cirrhosis); (V) previous use of ARNI; (VI) history of allergy or contraindications to the study drugs 


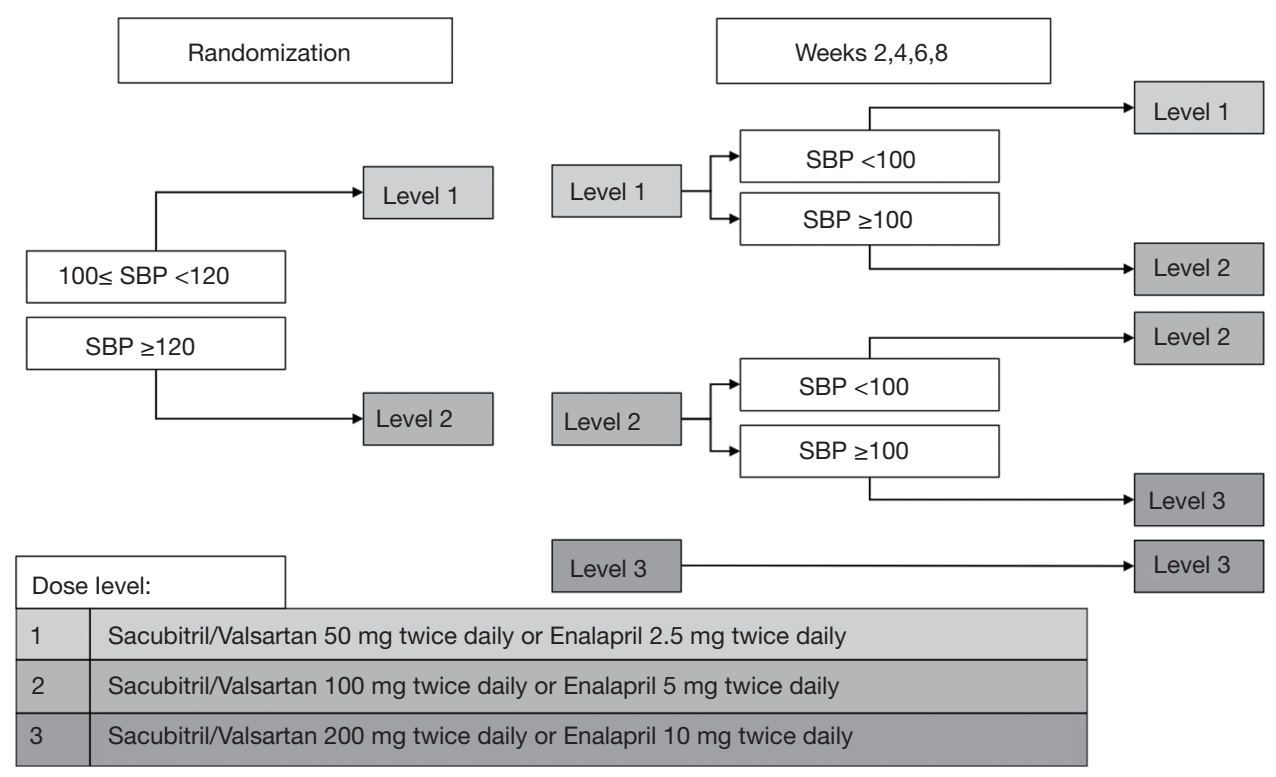

Figure 1 Dose titration algorithm. SBP, systolic blood pressure.

including ARNI, ACEI, and angiotensin receptor blocker (ARB); and (VII) life expectancy $<1$ year.

\section{Study procedure}

After the primary PCI operation, patients were admitted to the coronary care unit (CCU) for continuous medical monitoring and specialist nursing. Drug therapy mainly included dual antiplatelet, statins, beta blockers and enalapril. Mineralocorticoid receptor antagonists (MRAs), diuretics, and inotropes were used according to the patient's condition. Within 24 hours after PCI, the patients were randomly divided (1:1) into 2 groups (ARNI/enalapril) using $\mathrm{R}$ software-based random functions. Patients randomized to the enalapril group continued to receive enalapril, while patients randomized to the ARNI group ceased to receive enalapril with a minimum 36-hour washout period prior to receiving the sacubitril/valsartan. An independent researcher generated the randomization sequence by using randomized permuted blocks and assigned the participants to the interventions. For allocation concealment, medications are placed in sequentially numbered, opaque, sealed containers. All study personnel and patients were blind to treatment allocation and had no way of influencing whether a participant would receive sacubitril/valsartan or enalapril. A dose titration algorithm was used to select the initial dose (sacubitril/valsartan $24 / 26$ or $49 / 51 \mathrm{mg}$ and enalapril 2.5 or $5 \mathrm{mg}$ ) at the beginning of hospitalization and subsequent dose (sacubitril/valsartan 97/103 mg and enalapril $10 \mathrm{mg}$ ) changes during the drug titration (Figure 1) (14). Patients had follow-ups at 4, 12, and 24 weeks after discharge from the hospital. This involved a blood test and echocardiograph.

\section{Echocardiography and laboratory assessment}

We used the MS-Fast N-terminal pro-B-type natriuretic peptide (NT-proBNP) automatic chemiluminescence immunoassay analyzer and supporting reagent card (Sophonix Co., Ltd., Beijing, China) to carry out NTproBNP quantitative detection. NT-proBNP levels were obtained from all patients before primary PCI and at 4, 12, and 24 weeks after discharge. Echocardiographic measurements were performed immediately after PCI and at 12 and 24 weeks with a Philips IE33 ultrasound system (Philips Medical System, Bothell, WA, USA). LV ejection fraction (LVEF), LV end-systolic volumes (LVESV), and LV end-diastolic volumes (LVEDV) were measured using the biplane Simpson method in the apical 4-chamber heart and 2-chamber heart view according to the recommendations issued by the American Society of Echocardiography and the European Cardiovascular Imaging Association (15).

\section{Endpoints and definitions}

The primary efficacy outcomes included differences 
between groups in change from baseline to week 4, 12, and 24 in NT-proBNP concentration as well as change from baseline to week 12 and 24 in LVEF, LVESV and LVEDV. Secondary outcomes were the time-to-first event analysis of the composite risk of death, reinfarction, outpatient HF or HF hospitalization, malignant arrhythmia, and stroke. HF hospitalization was defined as a patient exhibiting new or worsening symptoms, objective evidence of HF, and the need for initiation or intensification of HF-specific treatment. Symptoms included at least 1 of the following: dyspnea, decreased exercise tolerance, fatigue, or other symptoms of worsened end-organ perfusion or volume overload. Objective evidence needed to consist of at least 2 physical examination findings or a combination of at least 1 physical examination finding and 1 laboratory criterion. Physical examination findings included peripheral edema, bloating or ascites (without any other underlying causes of hepatic disease), pulmonary rales/crackles/crepitations, jugular venous pressure elevation and/or hepatojugular reflux, S3 gallop, or rapid body weight gain due to fluid retention. The main laboratory evidence included increased brain natriuretic peptide (BNP)/NT-proBNP concentration consistent with decompensation of $\mathrm{HF}$ (such as BNP $>500 \mathrm{pg} / \mathrm{mL}$ or NT-proBNP $>2,000 \mathrm{pg} / \mathrm{mL}$ ). Outpatient HF was defined as an emergent/unscheduled visit to an emergency department/outpatient clinic or a non-emergent visit for the primary diagnosis of HF (which did not require an overnight stay in a hospital ward). Malignant arrhythmia was defined as a composite event, including cardiac arrest, persistent ventricular tachycardia, and ventricular fibrillation. Safety outcomes were the incidence of worsening renal function, hypotension, hyperkalemia angioedema, and cough.

\section{Statistical analysis}

All data analyses were completed using $\mathrm{R}$ software version 3.6.1 (https://www.r-project.org/) and Empower (http:// www.empowerstats.com; X\&Y Solutions, Inc., Boston, MA, USA). To evaluate the primary and secondary outcomes, we used per-protocol (PP) analyses. The targeted sample size was mainly driven by the LVEF of the primary outcomes. With an assumed increase in LVEF of $3 \% \pm 6 \%$, the patients were included in a ratio $1: 1$ if $\alpha=0.05$, the test power was $80 \%$, and the sample size for each group was predicted to be 64 cases. Outcome variables were tested for normality using the Shapiro-Wilk test. Continuous variables are expressed using mean and standard deviation (SD) or geometric means and 95\% CI. Categorical variables are expressed in frequency and percentage. Comparison of baseline characteristics was carried out by using 2 -sample $t$-test and Fisher's exact test. The 2-sided significance level for all final tests was set to 0.05 . The change in the primary outcomes was tested suing a covariance analysis model adjusted for baseline values. Logarithmic transformation was used for NT-proBNP, due to its skewed distribution. Secondary outcomes data were evaluated suing Kaplan-Meier method and Cox proportional hazards models. The hazard ratio (HR), 95\% CI, and 2-sided $\mathrm{P}$ values were calculated using the model to adjust the following baseline prognostic factors: age, gender, body mass index, total cholesterol, triglycerides, history of hypertension, diabetes, and previous history.

\section{Results}

\section{Study participants and follow-up}

Among the 171 participants screened between February 17, 2019 and December 28, 2019, 131 patients met all the inclusion and exclusion criteria and were randomized (Figure 2). The baseline characteristics of the patients are shown in Table 1. In the total study population, the mean age was 60.3 (SD, 9.9) years, $104(79.4 \%)$ were males, $62(47.3 \%)$ had a history of hypertension, $33(25.2 \%)$ had a history of diabetes, and $50(38.2 \%)$ had previously received an ACEI or ARB treatment. The average time from symptom onset to balloon inflation was 3.2 (SD, 1.2) hours. Furthermore, 64 patients in the ARNI group and 64 patients in the enalapril group completed a laboratory examination and cardiac ultrasound during follow-up; 52 $(81.3 \%)$ patients in the enalapril group and $48(75.0 \%)$ in the ARNI group successfully titrated to the target dose.

\section{Study outcomes}

After treatment, NT-proBNP levels were significantly reduced in both groups from the baseline to week 24 . However, the NT-proBNP concentration of the ARNI group had decreased more significantly than had that of the enalapril group at each follow-up time (4 weeks: ratio of ARNI vs. enalapril 0.36, $95 \%$ CI: 0.24 to 0.52 , $\mathrm{P}<0.001 ; 12$ weeks: $0.54,95 \%$ CI: 0.35 to $0.79, \mathrm{P}<0.001$; and 24 weeks: $0.53,95 \% \mathrm{CI}: 0.32$ to $0.83, \mathrm{P}<0.001$; Figure $3 \mathrm{~A}$ and Table 2). Compared with the enalapril group, the ARNI group also had a significant reduction in LVEDV 


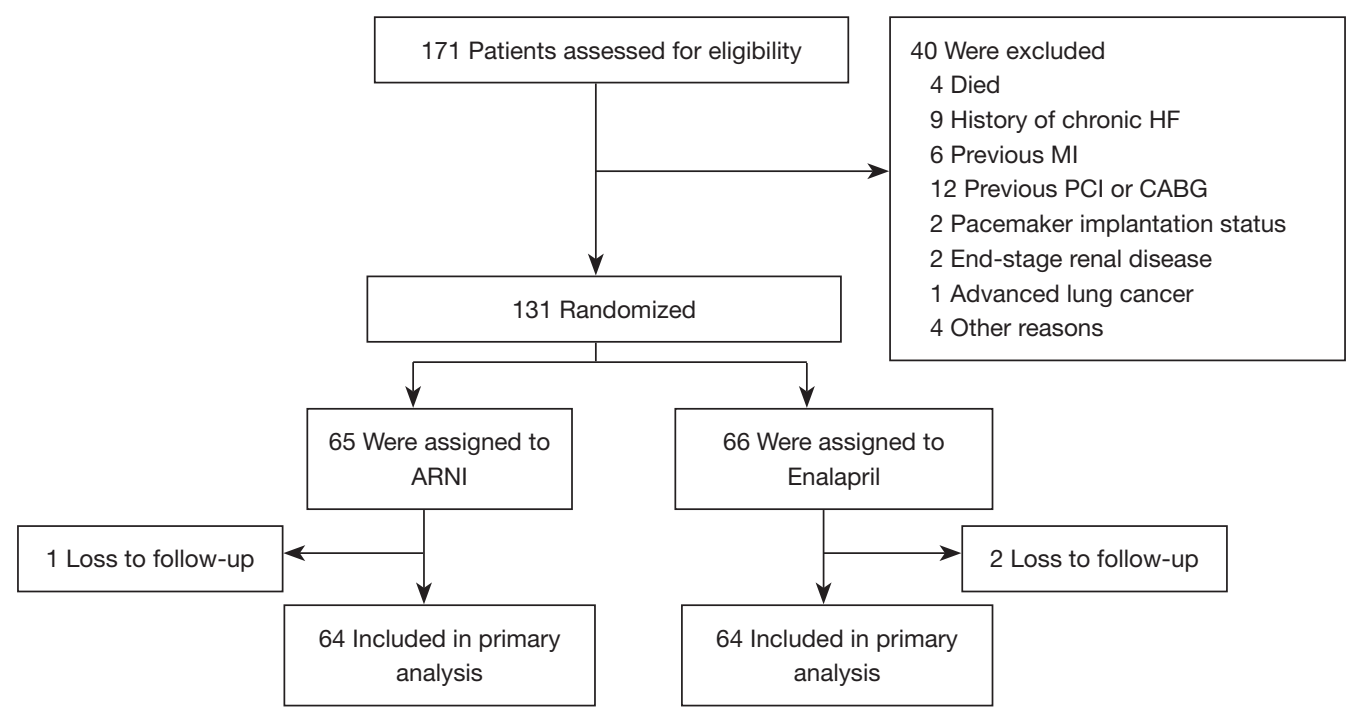

Figure 2 Study flow diagram. HF, heart failure; MI, myocardial infarction; PCI, percutaneous coronary intervention; CABG, coronary artery bypass grafting; ARNI, angiotensin receptor-neprilysin inhibitor.

(ARNI: from $124.55 \pm 21.4$ to $114.36 \pm 21.3 \mathrm{~mL}$; enalapril: from $121.08 \pm 21.2$ to $129.30 \pm 19.0 \mathrm{~mL}$; difference $-15.41 \mathrm{~mL}$; 95\% CI: -17.27 to $-13.54 \mathrm{~mL} ; \mathrm{P}<0.001)$ and LVESV (ARNI: from $69.06 \pm 22.5$ to $55.30 \pm 18.8 \mathrm{~mL}$; enalapril: from $65.00 \pm 20.0$ to $64.23 \pm 16.0 \mathrm{~mL}$; difference $-11.5 \mathrm{~mL} ; 95 \%$ CI: -16.06 to $-6.94 \mathrm{~mL} ; \mathrm{P}<0.001)$. In addition, at 24 weeks the ARNI group also had an improvement in LVEF (ARNI: from $45.75 \% \pm 9.7 \%$ to $51.77 \% \pm 8.8 \%$; enalapril: from $46.97 \% \pm 9.1 \%$ to $50.59 \% \pm 8.5 \%$; difference $3.39 \%$; $95 \%$ CI: $1.12 \%$ to $6.66 \% ; \mathrm{P}=0.011$; Figure $3 B-3 D$, and Table 3$)$.

After a median follow-up of 261 (interquartile range: 226 to 311) days, secondary outcomes (death, reinfarction, outpatient HF or HF hospitalization, malignant arrhythmia, or stroke) occurred in 13 participants $(20.3 \%)$ in the sacubitril/valsartan group and 22 participants (34.4\%) in the enalapril group. When compared with the enalapril group, the HR for secondary outcomes in the ARNI group was 0.56 (95\% CI: 0.28 to 1.12; $\mathrm{P}=0.102$; Figure $4 A$ and Table 4). Outpatient HF or HF hospitalization occurred in 6 ARNI patients (2 outpatient HF and 4 HF hospitalization, 9.4\%) compared to 17 enalapril patients (8 outpatient $\mathrm{HF}$ and 9 HF hospitalization, $26.6 \%$; HR 0.36; $95 \%$ CI: 0.14 to 0.94 ; $\mathrm{P}=0.037$; Figure $4 B$ and Table 4). The effect of ARNI on this outcome was consistent in an unadjusted analysis (HR 0.35; 95\% CI: 0.14 to $0.89 ; \mathrm{P}=0.027$ ).

During the treatment period, the incidence of hypotension in the ARNI group was higher than that in enalapril group, but the difference between the 2 groups was not significant $(18.8 \%$ vs. $7.8 \%, \mathrm{P}=0.068)$. No angioedema was found in the 2 groups. The incidence of worsening renal function, hyperkalemia, and cough was low, and there was no difference between the 2 groups (Table 5).

\section{Discussion}

This prospective, randomized, double-blind study was conducted to examine and compare the effect of early initiation ARNI and enalapril in patients with acute anterior STEMI after PCI. The most important findings of this trial were the following: (I) the timely application of ARNI led to a greater reduction in NT-proBNP than did enalapril; (II) compared to enalapril, ARNI was associated with an improvement in echocardiographic parameters including LVEF, LVESV, and LVEDV after 24 weeks; (III) ARNI was superior to enalapril in reducing the risk of outpatient HF or HF hospitalization; and (IV) ARNI had the tendency to increase the occurrence of hypotension, but it was well tolerated by most patients.

After AMI, myocardial mechanical stretch, secondary to $\mathrm{LV}$ dysfunction, is considered as one of the most important stimuli contributing to the increase of NT-proBNP (16). We observed a relatively rapid reduction of NT-proBNP concentration, apparent as early as 4 weeks, in patients with ARNI. The pharmacological action of the drug is not entirely clear, but possible mechanisms are outlined as follows: (I) compared with renin-angiotensin-aldosterone 
Table 1 Baseline characteristics of the study population

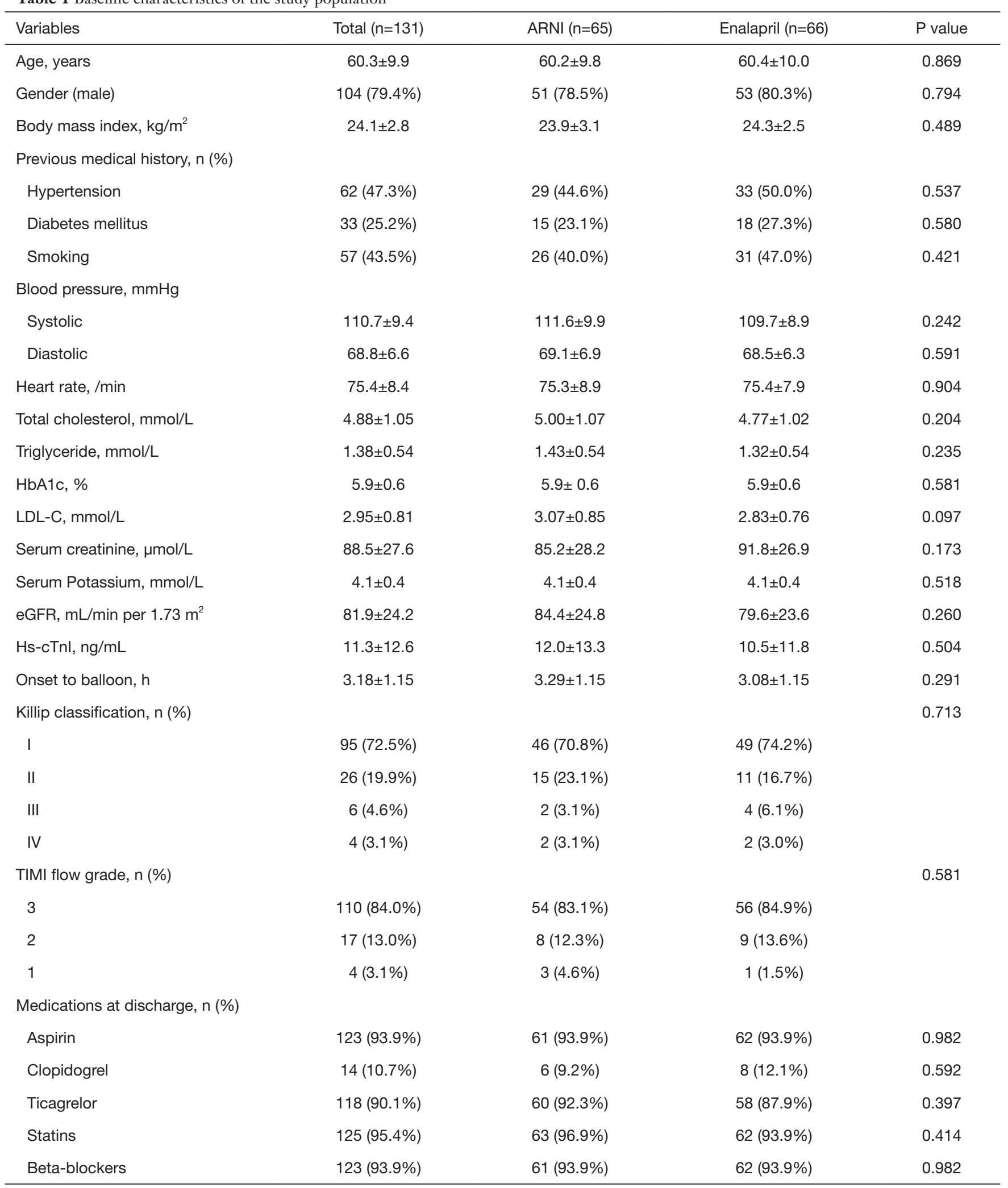

Table 1 (continued) 
Table 1 (continued)

\begin{tabular}{|c|c|c|c|c|}
\hline Variables & Total $(n=131)$ & ARNI $(n=65)$ & Enalapril $(n=66)$ & $P$ value \\
\hline Diuretics & $41(31.3 \%)$ & $19(29.2 \%)$ & 22 (33.3\%) & 0.613 \\
\hline Digoxin & $4(3.1 \%)$ & $2(3.1 \%)$ & $2(3.0 \%)$ & 0.988 \\
\hline
\end{tabular}

Data are presented as $n(\%)$ or the mean \pm SD. HbA1c, hemoglobin A1c; LDL-C, low-density lipoprotein cholesterol; eGFR, estimated glomerular filtration rate; Hs-cTnl, high sensitivity cardiac Troponin I; TIMI, thrombolysis in myocardial infarction; MRA, mineralocorticoid receptor antagonist.
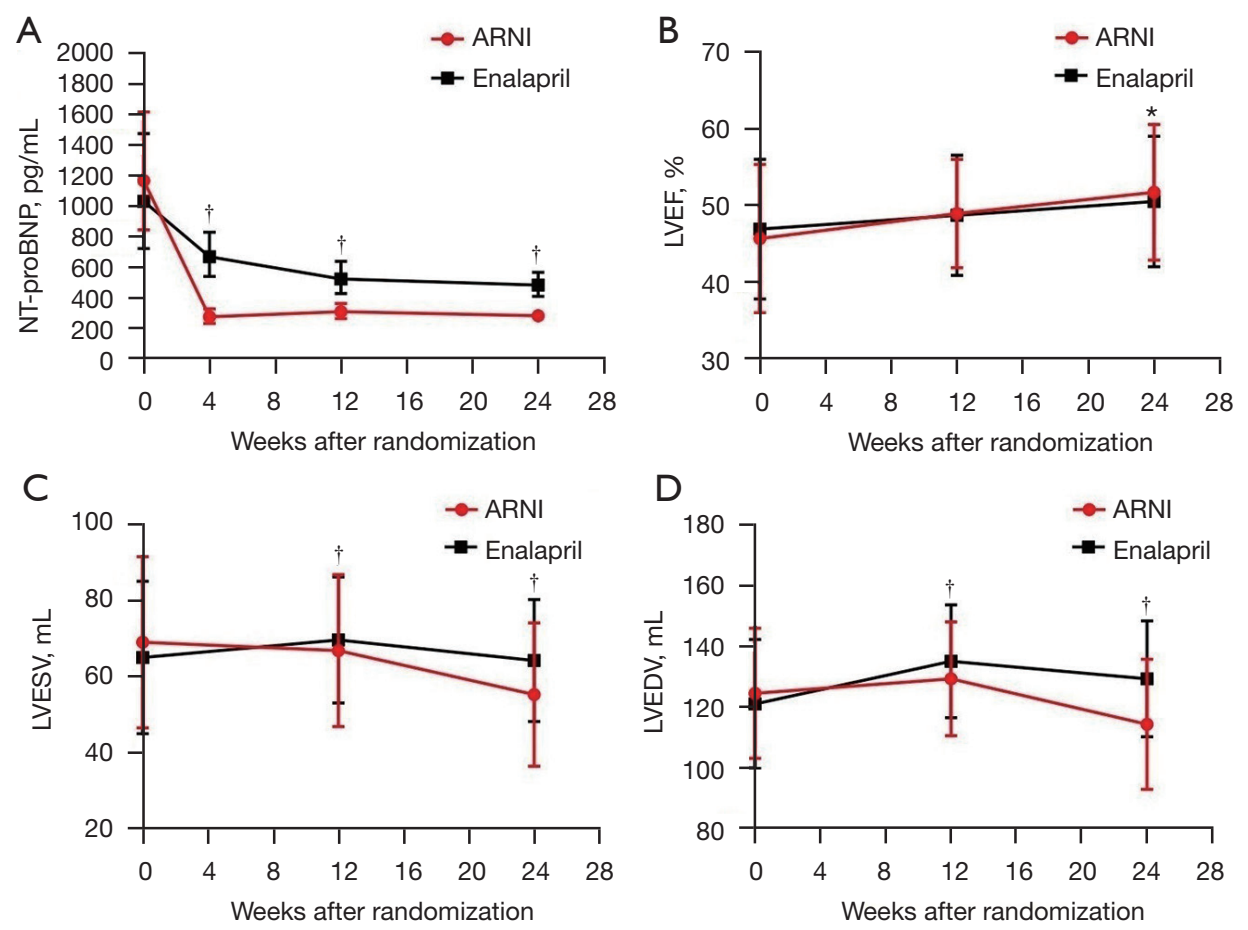

Figure 3 NT-proBNP (A) at baseline, 4, 12 and 24 weeks in the ARNI and Enalapril groups; LVEF (B), LVESV (C), and LVEDV (D) at baseline, 12, and 24 weeks in the ARNI and enalapril groups. *, $\mathrm{P}<0.05$ compared with the enalapril group; ${ }^{\dagger}, \mathrm{P}<0.001$ compared with the enalapril group; ARNI, angiotensin receptor-neprilysin inhibitor; LVEF, left ventricular ejection fraction; LVESV, left ventricular endsystolic volume; LVEDV, left ventricular end-diastolic volume.

system (RAAS) inhibitors, early application with ARNI after AMI might have a cardioprotective effect through the further inhibition of the expression and release of inflammation-associated cytokines in the infarcted area (11); (II) the natriuretic, diuretic, and vasodilating effects of natriuretic peptides (NPs) lead to a decrease in pre- and after-load, thereby inhibiting ventricular wall tension and ventricular dilatation in the early stage of AMI; and (III) by inhibiting the angiotensin II receptor, ARNI inhibits the expression of pro-cardiac hypertrophy and fibrosis factors, such as soluble suppression of tumorigenesis-2 (sST2) (17), matrix metalloproteinase 9 (MMP9) (11) and transforming growth factor $\beta$ (TGF- $\beta$ ) (18). ARNI can also lower the expression of $\beta$-myosin heavy chain ( $\beta$-MHC) genes (19), as well as down-regulate the expression of exosomal miR181a (regulates cell proliferation and apoptosis) (20) and connective tissue growth factor (CTGF, a strong marker pro-fibrotic remodeling) (19). Moreover, in animal experiments $(10,19)$, sacubitril/valsartan was found to improve the blood supply of the infarcted myocardium 
Table 2 Change in NT-proBNP (pg/mL) from baseline to 4, 12 and 24 weeks

\begin{tabular}{lccc}
\hline Variables & ARNI & Enalapril & $\begin{array}{c}\text { Ratio of ARNI vs. Enalapril, } \\
\text { geometric mean (95\% Cl) }\end{array}$ \\
\hline Baseline & $1,168[845-1,616]$ & $1,033[724-1,474]$ & \\
4 weeks & $279[236-331]$ & $671[542-831]$ & \\
Ratio of geometric means, & $0.24(0.20-0.29)$ & $0.65(0.55-0.77)$ & $0.36(0.24-0.52)$ \\
4 weeks/baseline $(95 \% \mathrm{Cl})$ & & & \\
12 weeks & $313[268-366]$ & $525[430-640]$ & $0.54(0.35-0.79)$ \\
$\begin{array}{l}\text { Ratio of geometric means, } \\
12 \text { weeks/baseline }(95 \% \mathrm{Cl})\end{array}$ & $0.27(0.21-0.34)$ & $0.51(0.42-0.62)$ & \\
24 weeks & $285[251-323]$ & $485[413-569]$ & $<0.001$ \\
$\begin{array}{l}\text { Ratio of geometric means, } \\
24 \text { weeks/baseline }(95 \% \mathrm{Cl})\end{array}$ & $0.24(0.19-0.31)$ & $0.47(0.37-0.59)$ & $0.53(0.32-0.83)$ \\
\hline
\end{tabular}

Data for NT-proBNP are geometric mean $(95 \% \mathrm{Cl})$. ARNI, angiotensin receptor-neprilysin inhibitor; $\mathrm{Cl}$, confidence interval.

and significantly attenuate the size of the LV scar after MI, whereas RAAS blocker were found to have no statistical significance in reducing the scar. It is widely acknowledged that NT-proBNP is an important predictor of adverse events after AMI (21-23). Heeschen et al. reported that in patients with high levels of NT-proBNP at baseline, the slow decline of NT-proBNP after myocardial injury was associated with a short-term adverse prognosis (22). Another study found a close correlation between the concentration of NT-proBNP and the long-term all-cause mortality and rate of rehospitalization for $\mathrm{HF}$ after MI (23). The results of 4 previous large-scale clinical trials showed that sacubitril/ valsartan reduced the NT-proBNP in patients with $\mathrm{HF}$ and the full-range of ejection fraction and acute decompensated HF $(9,14,24,25)$. A similar effect was observed with ARNI in this acute anterior STEMI population, suggesting that sacubitril/valsartan may provide a beneficial prognosis in patients with this disorder.

In the analyses of echocardiographic parameters, treatment with sacubitril/valsartan was associated with improvements in LVEF, as well as a lower LVESV and LVEDV. Our results are in line with those in many other animal and clinical studies $(10,12,19)$ and suggest the superiority of ARNI in cardiac remodeling. Compared with a single RAAS blocker, the improvement of cardiac ultrasound parameters observed with ARNI may be attributed to an increase in circulating NP levels. Previous clinical and animal studies have shown that the administration of NPs can provide cardioprotection and improve cardiac function and hemodynamic parameters (26).
In this study of acute anterior STEMI patients who underwent primary PCI, the inhibition of both the angiotensin II receptor and neprilysin was more effective in reducing the risk of outpatient $\mathrm{HF}$ or HF hospitalization than was ACE inhibition. However, the recently reported PARADISE-MI study (13) showed that compared with enalapril, ARNI failed to further reduce the combined events of cardiovascular death, HF hospitalization, and outpatient HF. Interestingly, when the primary endpoint was set as the total (first and recurrent) event adjudicated by the CEC, the rate ratio of the primary endpoint was 0.79 (95\% CI: 0.65 to $0.97 ; \mathrm{P}=0.02$ ), and when considering the use of the primary endpoint reported by the investigator, the overall HR was 0.85 (95\% CI: 0.75 to $0.96 ; \mathrm{P}=0.01$ ) while the HR for outpatient HF was 0.69 (95\% CI: 0.54 to $0.88 ; \mathrm{P}=0.003)$. The results of the PARADISE-MI trial were unexpected, but reasonable. At present, guideline-directed medical therapy (GDMT), which includes early reperfusion therapy and dual antiplatelet, statins, $\beta$-receptor blockers, and ACEI/ARB as its core methods, has reduced the 3-year mortality rate in AMI patients with $\mathrm{LV}$ insufficiency from $30 \%$ to $10 \%$ or less. It is indeed very difficult for ARNI to further obtain superior results on the basis of this optimized treatment. Unlike in the PARADISE-MI study, the patients included in the present trial were those with acute anterior STEMI, whose baseline NT-proBNP was at a relatively high level (geometric mean $1,168 \mathrm{pg} / \mathrm{mL}$ ). There are some signs that reversal of $L V$ remodeling with ARNI may be requisite on elevated LV wall stress (such as a high concentration of NT-proBNP at baseline) $(9,27,28)$. 


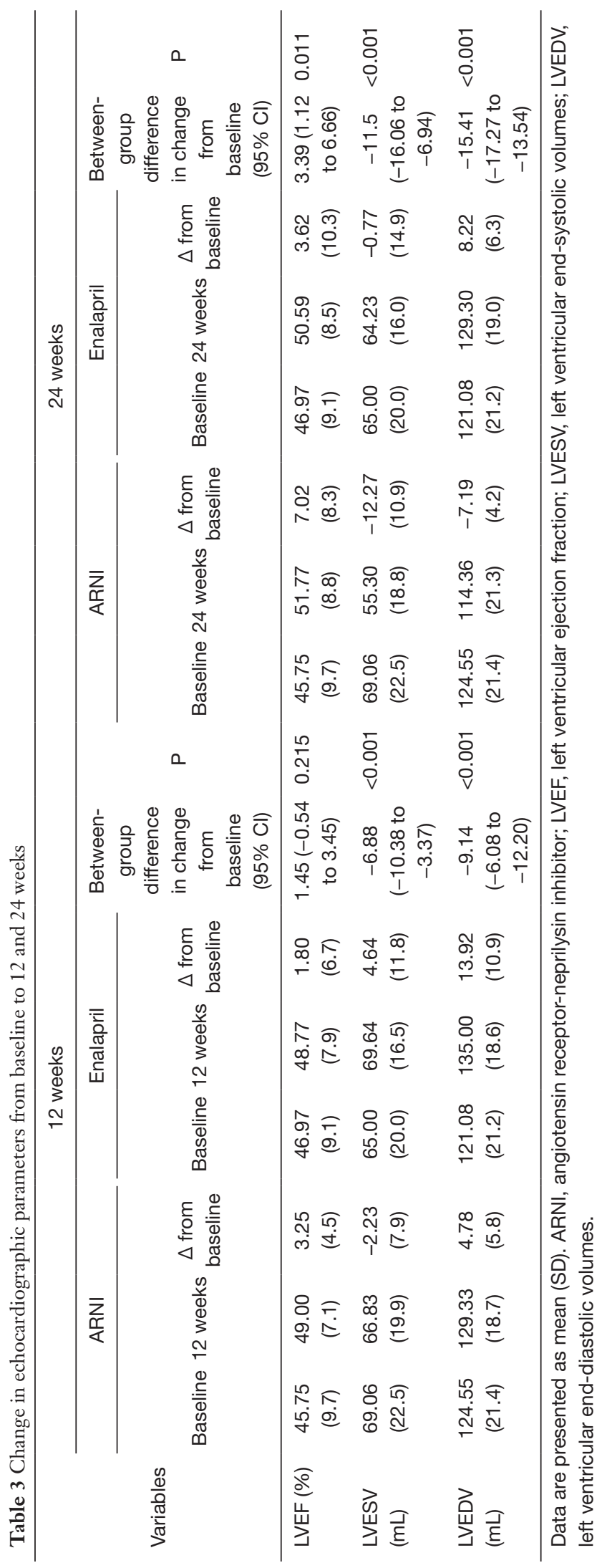

This may explain why the findings of this study saw ARNI significantly reduce the cumulative risk of outpatient HF or HF hospitalizations. Furthermore, there was no statistically significant difference in the overall incidence of cardiovascular events between the 2 groups, which may be due to the follow-up time or sample size being insufficiently long or large, respectively. However, the benefit of sacubitril/valsartan, which were also apparent in this study, could be seen in patients who had already received all other drugs known to improve prognosis among STEMI patients.

Our research shows that sacubitril/valsartan is well tolerated. Very few patients stopped the study drug, and $75 \%$ of the ARNI patients were successfully titrated to the target dose. The PARADIGM-HF (8) trial reported that the incidence of hypotension in the ARNI patients was significantly higher than that of the enalapril patients. In our study, ARNI also had the tendency to increase the occurrence of hypotension, which may be due to its powerful vasodilator effect. Although the greater hypotensive effect of ARNI may impair renal perfusion, there were no statistically significant differences in the incidence of deterioration of renal function or hyperkalemia between the 2 groups in our study. Contrary to concerns surrounding ARNI, several studies have shown that it can delay renal function decline and slow down the eGFR decline $(29,30)$. Therefore, based on current research, ARNI is more suitable for HF patients with renal insufficiency. In addition to, no angioedema occurred during the treatment, the incidence of cough was low, and there was no difference between the groups.

We used a per protocol analysis and therefore only data from patients with complete follow-up were analyzed. An intention-to-treat analysis would make the intervention less effective if patients assigned to the intervention group were lost to follow-up after randomization (31). With regard to follow-up, we do not expect a large difference in outcomes between a per-protocol analysis and an intention-to-treat analysis because of the number of patients lost to followup was low in our study. In summary, we found that the use of ARNI in patients with acute anterior STEMI is safe and effective, which indicates that treatment with sacubitril/ valsartan in the early stages of myocardial remodeling after AMI will provide greater benefit for patients.

\section{Limitations}

This study has the following limitations: (I) the patients we included did not include all types of AMI patients, but only 
A Secondary outcomes

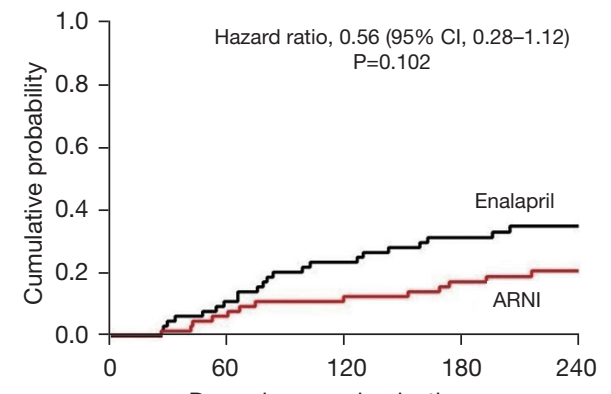

No. at risk

ARNI

Enalapril
Days since randomization

$\begin{array}{lllll}64 & 60 & 56 & 53 & 51 \\ 64 & 57 & 49 & 44 & 42\end{array}$

B

Outpatient HF or HF hospitalization

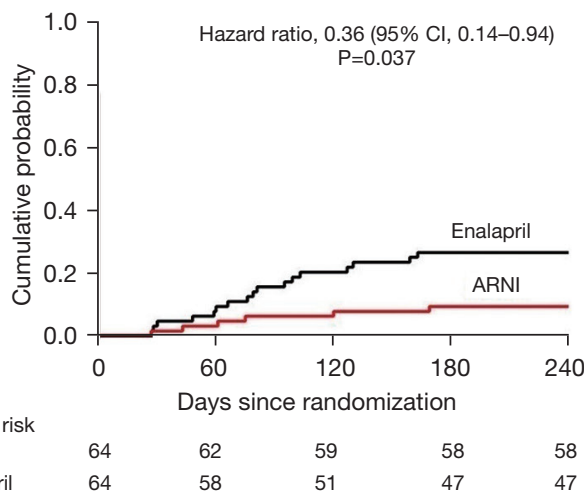

Figure 4 Cumulative Kaplan-Meier estimates of rates of secondary outcomes (according to study group. The hazard ratios for ARNI vs. enalapril are shown for the composites of death, reinfarction, outpatient HF or HF hospitalization, malignant arrhythmia, and stroke (A), HF or HF hospitalization (B). ARNI, angiotensin receptor-neprilysin inhibitor.

Table 4 Secondary outcomes

\begin{tabular}{|c|c|c|c|c|c|}
\hline Variables & Events, n (\%) & \multicolumn{2}{|c|}{ Adjusted } & \multicolumn{2}{|c|}{ Unadjusted } \\
\hline \multicolumn{6}{|c|}{ Composite outcomes } \\
\hline Enalapril & $22(34.4 \%)$ & Ref. & & Ref. & \\
\hline ARNI & $13(20.3 \%)$ & $0.56(0.28,1.12)$ & 0.102 & $0.53(0.27,1.06)$ & 0.071 \\
\hline Enalapril & $0(0.0 \%)$ & Ref. & & Ref. & \\
\hline ARNI & $1(1.6 \%)$ & Inf. (0.00, inf) & 1.000 & Inf. (0.00, inf) & 0.999 \\
\hline \multicolumn{6}{|c|}{ Outpatient HF or HF hospitalization } \\
\hline Enalapril & $17(26.6 \%)$ & Ref. & & Ref. & \\
\hline Enalapril & $3(4.7 \%)$ & Ref. & & Ref. & \\
\hline ARNI & $3(4.7 \%)$ & $0.84(0.14,5.04)$ & 0.848 & $1.00(0.20,4.95)$ & 0.999 \\
\hline \multicolumn{6}{|c|}{ Malignant arrhythmia } \\
\hline Enalapril & $4(6.3 \%)$ & Ref. & & Ref. & \\
\hline ARNI & $2(3.1 \%)$ & $0.29(0.04,2.26)$ & 0.236 & $0.48(0.09,2.62)$ & 0.396 \\
\hline \multicolumn{6}{|l|}{ Stroke } \\
\hline Enalapril & $1(1.6 \%)$ & Ref. & & Ref. & \\
\hline ARNI & $2(3.1 \%)$ & $2.21(0.15,31.81)$ & 0.561 & $2.00(0.18,22.04)$ & 0.572 \\
\hline
\end{tabular}

Adjusted results were adjusted for prespecified baseline characteristics: age, gender, body mass index, total cholesterol, triglycerides, history of hypertension, diabetes and previous smoking. ARNI, angiotensin receptor-neprilysin inhibitor. 
Table 5 Safety outcomes

\begin{tabular}{lccc}
\hline Variables & ARNI $(\mathrm{n}=64)$ & Enalapril $(\mathrm{n}=64)$ & $\mathrm{P}$ value \\
\hline All events & $16(25.0 \%)$ & $17(26.6 \%)$ & 0.840 \\
Worsening renal function & $3(4.7 \%)$ & $6(9.4 \%)$ & 0.300 \\
Hypotension & $12(18.8 \%)$ & $5(7.8 \%)$ & 0.068 \\
Hyperkalemia & $3(4.7 \%)$ & $4(6.2 \%)$ & 0.697 \\
Cough & $1(1.6 \%)$ & $3(4.7 \%)$ & 0.310 \\
\hline
\end{tabular}

Data are presented as $\mathrm{n}(\%)$. ARNI, angiotensin receptor-neprilysin inhibitor.

acute anterior STEMI patients, with the culprit vessel being the anterior descending branch, and thus the efficacy of sacubitril/valsartan in other types of MI therefore requires further exploration; (II) this study only measured the changes in NT-proBNP, but not many other biomarkers related to neprilysin inhibition, neurohumoral activation, or cardiac remodeling; and (III) the data from this study were derived from a small sample at a single-center, and thus further verification will be needed with data from future studies using multiple centers and large samples.

\section{Conclusions}

For patients with acute anterior STEMI undergoing primary PCI, we found that when compared with enalapril, ARNI significantly decreases the concentration of NTproBNP, improves LV systolic function, and reduces the risk of outpatient HF or HF hospitalization.

\section{Acknowledgments}

The authors would like to thank Chao Li (Autonomous University of Madrid) for technical assistance. The authors also thank the editor J. Goetz and J. Gray in AME Editing Service for their help in language editing. The authors particularly acknowledge the patients for their participation. Funding: This work was supported by Science and Technology Research Project of Jiangxi Provincial Department of Education (No. GJJ200185).

\section{Footnote}

Reporting Checklist: The authors have completed the CONSORT reporting checklist. Available at https://cdt. amegroups.com/article/view/10.21037/cdt-21-386/rc

Trial Protocol: Available at https://cdt.amegroups.com/ article/view/10.21037/cdt-21-386/tp

Data Sharing Statement: Available at https://cdt.amegroups. com/article/view/10.21037/cdt-21-386/dss

Peer Review File: Available at https://cdt.amegroups.com/ article/view/10.21037/cdt-21-386/prf

Conflicts of Interest: All authors have completed the ICMJE uniform disclosure form (available at https://cdt.amegroups. com/article/view/10.21037/cdt-21-386/coif). The authors have no conflicts of interest to declare.

Ethical Statement: The authors are accountable for all aspects of the work in ensuring that questions related to the accuracy or integrity of any part of the work are appropriately investigated and resolved. The study was conducted in accordance with the Declaration of Helsinki (as revised in 2013), and the Ethical Guidelines for Medical and Health Research Involving Human Subjects. This study was approved by the Ethics Committee of the Second Affiliated Hospital of Nanchang University (No. 2019005) and informed consent was taken from all the patients

Open Access Statement: This is an Open Access article distributed in accordance with the Creative Commons Attribution-NonCommercial-NoDerivs 4.0 International License (CC BY-NC-ND 4.0), which permits the noncommercial replication and distribution of the article with the strict proviso that no changes or edits are made and the original work is properly cited (including links to both the formal publication through the relevant DOI and the license). See: https://creativecommons.org/licenses/by-nc-nd/4.0/.

\section{References}

1. van der Bijl P, Abou R, Goedemans L, et al. Left 
Ventricular Post-Infarct Remodeling: Implications for Systolic Function Improvement and Outcomes in the Modern Era. JACC Heart Fail 2020;8:131-40.

2. Burchfield JS, Xie M, Hill JA. Pathological ventricular remodeling: mechanisms: part 1 of 2 . Circulation 2013;128:388-400.

3. Huttin O, Coiro S, Selton-Suty C, et al. Prediction of Left Ventricular Remodeling after a Myocardial Infarction: Role of Myocardial Deformation: A Systematic Review and Meta-Analysis. PLoS One 2016;11:e0168349.

4. Zaliaduonyte-Peksiene D, Simonyte S, Lesauskaite V, et al. Left ventricular remodelling after acute myocardial infarction: impact of clinical, echocardiographic parameters and polymorphism of angiotensinogen gene. J Renin Angiotensin Aldosterone Syst 2014;15:286-93.

5. Tarantini G, Napodano M, Gasparetto N, et al. Impact of multivessel coronary artery disease on early ischemic injury, late clinical outcome, and remodeling in patients with acute myocardial infarction treated by primary coronary angioplasty. Coron Artery Dis 2010;21:78-86.

6. Wu E, Ortiz JT, Tejedor P, et al. Infarct size by contrast enhanced cardiac magnetic resonance is a stronger predictor of outcomes than left ventricular ejection fraction or end-systolic volume index: prospective cohort study. Heart 2008;94:730-6.

7. Hubers SA, Brown NJ. Combined Angiotensin Receptor Antagonism and Neprilysin Inhibition. Circulation 2016;133:1115-24.

8. McMurray JJ, Packer M, Desai AS, et al. Angiotensinneprilysin inhibition versus enalapril in heart failure. $\mathrm{N}$ Engl J Med 2014;371:993-1004.

9. Desai AS, Solomon SD, Shah AM, et al. Effect of Sacubitril-Valsartan vs Enalapril on Aortic Stiffness in Patients With Heart Failure and Reduced Ejection Fraction: A Randomized Clinical Trial. JAMA 2019;322:1077-84.

10. Torrado J, Cain C, Mauro AG, et al. Sacubitril/Valsartan Averts Adverse Post-Infarction Ventricular Remodeling and Preserves Systolic Function in Rabbits. J Am Coll Cardiol 2018;72:2342-56.

11. Ishii M, Kaikita K, Sato K, et al. Cardioprotective Effects of LCZ696 (Sacubitril/Valsartan) After Experimental Acute Myocardial Infarction. JACC Basic Transl Sci 2017;2:655-68.

12. Kompa AR, Lu J, Weller TJ, et al. Angiotensin receptor neprilysin inhibition provides superior cardioprotection compared to angiotensin converting enzyme inhibition after experimental myocardial infarction. Int J Cardiol 2018;258:192-8.

13. Pfeffer M. Prospective ARNI versus ACE inhibitor trial to determine superiority in reducing heart failure events after myocardial infarction (PARADISE-MI). Presented at: ACC 2021. May 15, 2021.

14. Velazquez EJ, Morrow DA, DeVore AD, et al. AngiotensinNeprilysin Inhibition in Acute Decompensated Heart Failure. N Engl J Med 2019;380:539-48.

15. Lang RM, Badano LP, Mor-Avi V, et al. Recommendations for cardiac chamber quantification by echocardiography in adults: an update from the American Society of Echocardiography and the European Association of Cardiovascular Imaging. J Am Soc Echocardiogr 2015;28:1-39.e14.

16. Peng B, Xia H, Ni A, et al. Serum NT-proBNP on admission can predict ST-segment resolution in patients with acute myocardial infarction after primary percutaneous coronary intervention. Herz 2015;40:898-905.

17. Zile MR, O'Meara E, Claggett B, et al. Effects of Sacubitril/Valsartan on Biomarkers of Extracellular Matrix Regulation in Patients With HFrEF. J Am Coll Cardiol 2019;73:795-806.

18. Suematsu Y, Miura S, Goto M, et al. LCZ696, an angiotensin receptor-neprilysin inhibitor, improves cardiac function with the attenuation of fibrosis in heart failure with reduced ejection fraction in streptozotocin-induced diabetic mice. Eur J Heart Fail 2016;18:386-93.

19. Pfau D, Thorn SL, Zhang J, et al. Angiotensin Receptor Neprilysin Inhibitor Attenuates Myocardial Remodeling and Improves Infarct Perfusion in Experimental Heart Failure. Sci Rep 2019;9:5791.

20. Vaskova E, Ikeda G, Tada Y, et al. Sacubitril/Valsartan Improves Cardiac Function and Decreases Myocardial Fibrosis Via Downregulation of Exosomal miR-181a in a Rodent Chronic Myocardial Infarction Model. J Am Heart Assoc 2020;9:e015640.

21. O'Donoghue ML, Morrow DA, Cannon CP, et al. Multimarker Risk Stratification in Patients With Acute Myocardial Infarction. J Am Heart Assoc 2016;5:002586.

22. Heeschen C, Hamm CW, Mitrovic V, et al. N-terminal pro-B-type natriuretic peptide levels for dynamic risk stratification of patients with acute coronary syndromes. Circulation 2004;110:3206-12.

23. Omland T. Clinical and laboratory diagnostics of cardiovascular disease: focus on natriuretic peptides and cardiac ischemia. Scand J Clin Lab Invest Suppl 2005;240:18-24. 
24. Solomon SD, Zile M, Pieske B, et al. The angiotensin receptor neprilysin inhibitor LCZ696 in heart failure with preserved ejection fraction: a phase 2 double-blind randomised controlled trial. Lancet 2012;380:1387-95.

25. Januzzi JL Jr, Prescott MF, Butler J, et al. Association of Change in N-Terminal Pro-B-Type Natriuretic Peptide Following Initiation of Sacubitril-Valsartan Treatment With Cardiac Structure and Function in Patients With Heart Failure With Reduced Ejection Fraction. JAMA 2019;322:1085-95.

26. Nishikimi T, Maeda N, Matsuoka H. The role of natriuretic peptides in cardioprotection. Cardiovasc Res 2006;69:318-28.

27. Lee MMY, Brooksbank KJM, Wetherall K, et al. Effect of Empagliflozin on Left Ventricular Volumes in Patients With Type 2 Diabetes, or Prediabetes, and Heart Failure With Reduced Ejection Fraction (SUGAR-DM-HF).

Cite this article as: Dong $\mathrm{Y}, \mathrm{Xu} \mathrm{Y,} \mathrm{Ding} \mathrm{C,} \mathrm{Yu} \mathrm{Z,} \mathrm{Yu} \mathrm{Z,} \mathrm{Xia}$ $\mathrm{X}$, Chen Y, Jiang X. Comparing the efficacy of angiotensin receptor-neprilysin inhibitor and enalapril in acute anterior STEMI patients after primary percutaneous coronary intervention: a prospective randomized trial. Cardiovasc Diagn Ther 2022;12(1):42-54. doi: 10.21037/cdt-21-386
Circulation 2021;143:516-25.

28. Docherty KF, Campbell RT, Brooksbank KJM, et al. Effect of Neprilysin Inhibition on Left Ventricular Remodeling in Patients With Asymptomatic Left Ventricular Systolic Dysfunction Late After Myocardial Infarction. Circulation 2021;144:199-209.

29. Damman K, Gori M, Claggett B, et al. Renal Effects and Associated Outcomes During AngiotensinNeprilysin Inhibition in Heart Failure. JACC Heart Fail 2018;6:489-98.

30. Mc Causland FR, Lefkowitz MP, Claggett B, et al. Angiotensin-Neprilysin Inhibition and Renal Outcomes in Heart Failure With Preserved Ejection Fraction. Circulation 2020;142:1236-45.

31. Hernán MA, Robins JM. Per-Protocol Analyses of Pragmatic Trials. N Engl J Med 2017;377:1391-8. 